\title{
Trinken beim Sport: individuell und zielorientiert
}

\author{
Uwe Schröder, Günter Wagner
}

\begin{abstract}
Eine an individuellen Schweißverlusten und der persönlichen sportlichen
Zielsetzung ausgerichtete Trinkstrategie vor, während und nach der Aktivität ist ein entscheidender Faktor, um die Leistungsfähigkeit beim Sport zu unterstützen und die Regeneration zu optimieren. Konkrete Trinkempfehlungen für sportlich Aktive haben individuelle physiologische Grundbedingungen, Umgebungsfaktoren und logistische Rahmenbedingungen zu berücksichtigen.
\end{abstract}

\section{Zu wenig und zu viel getrunken schränkt Leistungsfähigkeit ein}

Ca. $65 \%$ des gesamten Körperwassers sind intrazellulär, $35 \%$ extrazellulär lokalisiert [1]. Insbesondere bei hohen Außen- oder Hallentemperaturen, bei hoher Luftfeuchtigkeit und bei sehr langen, intensiven Aktivitäten kann sich dieses Verhältnis durch sportbedingte Schweißverluste drastisch ändern. Daraus können sowohl bei einer Hypo- als auch bei einer, meist auf Verhaltensfehlern basierenden, Hyperhydratation ernsthafte gesundheitliche Beeinträchtigungen resultieren ( $\triangleright$ Abb. 1) [2][3]. Grundsätzlich sollte während des Trainings und im Wettkampf nicht mehr getrunken werden als ausgeschwitzt wird. Ist das Körpergewicht nach dem Sport höher als zu Sportbeginn, wurde zu viel getrunken. Dieses als „Overdrinking“ bezeichnete Phänomen ist die Hauptursache für eine kritische Natrium-Blutkonzentration von weniger als

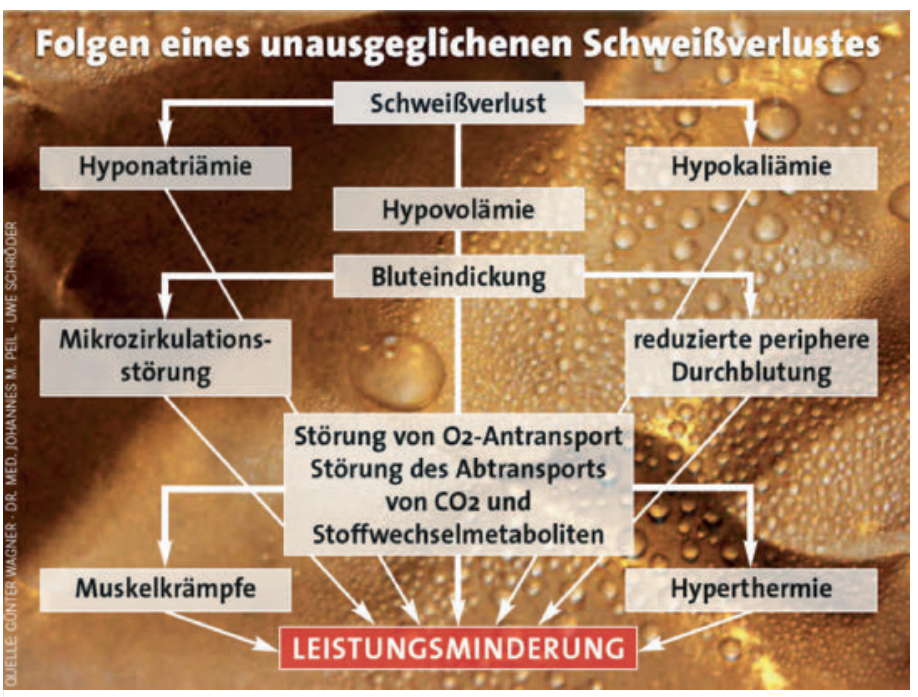

Abb. 1 Folgen eines unausgeglichenen Schweißverlustes.
135 mmol Na/Liter Blut während der Aktivität [4][5][6]. Seit 1985 wurden weltweit 14 Todesfälle durch eine sportbedingte Hyponatriämie (EAH = Exercise-associated Hyponatremia) dokumentiert [7].

\section{PRAXISTIPP}

Dem Risiko des Overdrinkings und der resultierenden Wasserintoxikation trägt die Empfehlung der International Marathon Medical Directors Association (IMMDA, 2018) Rechnung, während eines Marathonlaufes nicht mehr als ca. $900 \mathrm{ml}$ pro Stunde zu trinken.

\section{Gut hydriert starten}

Vor dem Start helfen natrium- bzw. kochsalzhaltige Getränke, das Wasser im Körper zu binden und gut hydriert zu starten. Es wird empfohlen, 4 Stunden vor Training/Wettkampf langsam ca. 5-7 ml salzhaltige Getränke pro kg Körpergewicht (KG) zu trinken, entsprechend 350-500 ml bei einem 70 kg schweren Sportler. Wird bis ca. 2 Stunden vor Belastungsbeginn kein Urin produziert oder ist dieser noch sehr dunkel, sollten weitere $3-5 \mathrm{ml} / \mathrm{kg}$ KG getrunken werden. So besteht ausreichend Zeit für die Urinausscheidung, um gut hydriert zu starten.

Merke
Das Ziel ist: Hypo- und Hyperhydratation vermeiden!

Während der Aktivität müssen Trinkmenge und Inhaltsstoffe des Getränks mit Intensität, Dauer und Art der sportlichen Aktivität korrespondieren, um die individuellen Sportziele effektiv zu unterstützen. Der Hydratationsstatus zu Beginn der Aktivität, die während des Sports zu erwartenden individuellen Schweißverluste und die Verträglichkeit sind ebenfalls zu berücksichtigen. Die individuel- 
le Trinkmenge ist so zu wählen, dass während des Sports Verluste der Körpermasse von mehr als $2 \%$ bei Hobbyathleten und bis $3 \%$ bei leistungsorientierten Sportlern vermieden werden. Parallel darf die Trinkmenge nicht zur Hyperhydratation führen [8].

Mit zunehmendem Flüssigkeitsdefizit werden sowohl die mentale als auch die physiologische Leistungsfähigkeit deutlich vermindert. Bei kurzen intensiven Belastungen ist die Leistungsfähigkeit durch Hypohydratation weit weniger beeinträchtigt als die Ausdauerleistungsfähigkeit. Sie kann vor allem bei weniger gut Trainierten bereits ab einem Wasserverlust von 2 \% des Körpergewichts eingeschränkt sein. Ab dieser Grenze steigt die Körperkerntemperatur. Die Herzleistung und damit die aerobe Leistung sinken [9][10][11].

\section{Merke}

Ein Wasserverlust von $2 \%$ des Körpergewichts kann die Ausdauerleistung bereits beeinträchtigen.

$2 \%$ entsprechen bei einem $75 \mathrm{~kg}$ schweren Sportler einem Schweißverlust von ca. 1,5 Liter, der aus intrazellulärem, interstitiellem und aus Wasser aus dem Blut resultiert.

Der Wasseranteil des menschlichen Körpers ist bezogen auf die fettfreie Masse recht konstant und beträgt ca. 74\%. Damit erklären sich hohe Wasserdefizite von $4 \%$ ohne Leistungseinschränkung bei Spitzenathleten. Ihre fettfreie Masse ist hoch, entsprechend hoch ist ihr „Wasserdepot“. Freizeitsportler hingegen weisen meist einen höheren Körperfett-, aber einen geringeren Muskelanteil und somit weniger verfügbares Körperwasser auf.

\section{PRAXISTIPP}

Schweißbedingte Wasserverluste von mehr als $5 \%$ des Körpergewichts schränken die Leistungsfähigkeit bis zu einem Drittel ein und sollten auch im Hochleistungssport grundsätzlich vermieden werden.

In Spielsportarten mit hoher kognitiver Beanspruchung kann es bereits bei geringeren Schweißverlusten zu Beeinträchtigungen des Leistungsniveaus kommen. Im Rahmen der Rosbacher Trinkstudien wurden bereits bei einem Wasserdefizit von nur $1 \%$ der Körpermasse verminderte mentale Leistungsparameter gemessen [12][13]. Daher sind die seit 2014 bestehenden Regeländerungen der Major Soccer League, der FIFA sowie bei den US Open im Tennis hinsichtlich hitzebedingter Trinkpausen auch zur Stabilisierung der mentalen Leistungsfähigkeit sinnvoll. Für sehr hohe Umgebungstemperaturen wurden im Fußball erst kürzlich längere Kühlpausen eingeführt [14]. 


\section{Belastungsempfinden steigt bei Hypohydratation}

Viele Sportler und Sportlerinnen beginnen ihr Training schlecht hydriert oder starten mit einem bereits bestehenden Wasserdefizit in Wettkämpfe. Unter diesen Bedingungen können bereits während Trainingseinheiten von weniger als einer Stunde Dauer ohne Getränkeaufnahme ein erhöhter Anstrengungsgrad und relevante Leistungseinschränkungen resultieren [4]. Bei Sportanfängern, Gesundheitssportlern und Aktiven mit der Zielsetzung Gewichtsreduktion führt dies oft zum vorzeitigen Abbruch der geplanten Aktivität. Ein frühzeitiger Ausgleich der Schweißverluste kann ein Fortsetzen der Aktivität durch geringeres Belastungsempfinden begünstigen und damit zum Erreichen der sportlichen/gesundheitsorientierten Zielsetzung beitragen.

\section{PRAXISTIPP}

Geschmack und Temperatur beeinflussen die Akzeptanz von Getränken und können so die Trinkmengen erhöhen und das Erreichen der Zieltrinkmengen erleichtern oder entsprechend bei geschmacklicher Nichtakzeptanz erschweren.

\section{Individuelle Schweißverluste bestimmen}

Die in Studien durchschnittlich gemessenen Schweißverluste variieren stark zwischen 0,5-1,9l/h [15]. Hitzeakklimatisierte Aktive weisen höhere Schweißraten und damit ein höheres Hypohydratationsrisiko auf [16]. Für alle regelmäßig und leistungsorientiert Aktiven sollte es daher selbstverständlich sein, den individuellen Wasserbedarf während und nach dem Sport sowie bei unterschiedlichen Umgebungsbedingungen zu kennen und entsprechend zu handeln. Um die individuellen Schweißverluste zu bestimmen, hat sich das Wiegen vor und nach der Aktivität als gesicherte, evidenzbasierte Maßnahme etabliert [17].

\section{LINK HYDRATATIONSTEST}

Ein Vordruck für einen Hydratationstest unter Berücksichtigung von Urinverlusten und Getränkeverzehr steht als Download unter www.dise.online zur Verfügung.

Unter Ruhe- und Alltagsbedingungen ist das Durstgefühl zur Regulation des individuellen Getränkekonsums meist ausreichend. Auch Aktiven wird empfohlen, während der Aktivität erst dann zu trinken, wenn sie Durst empfinden - ein Sportbeginn im gut hydrierten Zustand vorausgesetzt [23]. Im Wettkampf kann das Durstempfinden allerdings wegen der Ausschüttung von Stresshormonen un- terdrückt werden. Auch bei Mannschaftssportarten, bei älteren Sportlern sowie bei Kindern und Jugendlichen ist das Durstempfinden während des Sports oft kein ausreichend valider Indikator, der die Stabilisierung des Wasserhaushalts sicherstellt [18]. Daher ist für sie ein „Trinken nach Plan" die empfehlenswerte Variante.

\section{Für Wasserausgleich sorgen}

In $>$ Abb. $\mathbf{2}$ ist grafisch dargestellt, wie man den Flüssigkeitsbedarf ermitteln kann, der durch Schweißverluste beim Sport entsteht. Ein $70 \mathrm{~kg}$ schwerer Athlet im dargestellten Beispiel müsste ein Volumen von 2,6 0 ,7 I trinken, um eine Dehydratation von $>2 \%$ zu verhindern, wenn 4 I Körperwasser beispielsweise während eines Marathons ausgeschwitzt werden. Bei kürzeren Aktivitäten, z. B. Laufstrecken bis $10 \mathrm{~km}$, sind Wasserverluste von mehr als $2 \%$ des Körpergewichts unwahrscheinlich. Daher müsste derselbe Athlet während des Sports keine Getränke aufnehmen [23].

Praxisbeispiel siehe $>$ Abb. 2.

\section{Schweiß ist mehr als Wasser}

Mit dem Schweiß werden relevante Mengen an Elektrolyten ausgeschieden, vorwiegend Natrium, Calcium und Magnesium sowie in geringen Mengen Kalium ( $\triangleright$ Tab. 1). Die Natriumkonzentration im Schweiß ist im Vergleich zur Blutnatriumkonzentration hypoton. Sie nimmt ab ca. 45 Minuten Sportdauer mit der Länge der Aktivität zu und beträgt durchschnittlich ca. 50 mmol/l, entsprechend ca. $1,8 \mathrm{~g} \mathrm{Salz/l}[4][19]$.

Wird bei geringen Schweißverlusten und kurzen Trainingseinheiten getrunken, ist aus den beschriebenen Gründen mineralstoffreiches Mineralwasser ausreichend. Zum Ausgleich hoher Schweißverluste bei hohen Lufttemperaturen und bei Aktivitäten von mehr als 120 Minuten sollten Getränke mindestens 400 mg Natrium/l enthalten [4][20]. Besonders „gesundheitsorientierte“ Ausdauersportler, die im Alltag ihre Salzaufnahme bewusst einschränken oder zusätzliche Schweißverluste z. B. durch regelmäßige Saunabesuche aufweisen, sollten gezielt auf den Natrium-/ Kochsalzgehalt des Sportgetränks achten [18][23].

\section{Energiequelle Mono- und Disaccharide}

Ist die Zielsetzung des Trainings, einen Reiz für den Fettstoffwechsel zu setzen, kann auf Kohlenhydrate im Getränk verzichtet werden. Um während intensiver Aktivitäten, z. B. beim Intervalltraining, in Ballsportarten oder während langer Ausdauereinheiten mit Wettkampfgeschwindigkeit, Energie bereitzustellen, sind schnellverfügbare Kohlenhydrate im Getränk jedoch sinnvoll. Die Europäische Behörde für Lebensmittelsicherheit (EFSA) hat 2011 zwei Health-Claims für Sportgetränke beim Ausdauersport verabschiedet: „Kohlenhydrat-Elektrolyt-Getränke erhöhen 
die Absorption von Wasser während körperlicher Anstrengung“ und „Kohlenhydrat-Elektrolyt-Getränke tragen zum Erhalt der Ausdauerleistung während längerem Ausdauertraining bei." Die Getränke sollen 80-350 kcal pro Liter aus Kohlenhydraten liefern (ca. 20-87 g). Davon müssen mindestens 75\% eine deutliche Wirkung auf den Blutzuckerspiegel auslösen. Mono- und Disaccharide sowie Maltodextrine sind somit als Energielieferanten gefordert. Weiterhin muss ein derartiges Sportgetränk nach EFSAVorgaben mindestens $460 \mathrm{mg}$ und maximal $1150 \mathrm{mg}$ Natrium pro Liter (ca. 1,2 g Kochsalz) enthalten. Zudem müssen die Getränke leicht hypoton oder mindestens isoton sein [20].

2018 hat die EFSA eine weitere Wirkaussage für Getränke zur Unterstützung spezifischer sportlicher Aktivitäten formuliert: „Kohlenhydratlösungen können zu einer Verbesserung der körperlichen Leistungsfähigkeit während intensiver und langer körperlicher Aktivität bei gesunden Erwachsenen beitragen“. Um den Effekt zu erzielen, sollten die Getränke zwischen 30 und $60 \mathrm{~g} \mathrm{KH/h}$ Glucose, Gemische aus Glucose und Fructose, Saccharose und/oder Maltodextrine enthalten. Bei sehr langen Aktivitäten (>2 h) sind bis zu $90 \mathrm{~g} \mathrm{KH} / \mathrm{h}$ sinnvoll. Hier sollte der Fructose-Anteil etwa ein Drittel der Gesamtkohlenhydrate ausmachen. Die Zielgruppe sind gesunde, trainierte Erwachsene bei mindestens 60-minütiger körperlicher Aktivität mit hoher Intensität (>65\% der $\mathrm{VO}_{2}$ max) [22].

\section{PRAXISTIPP}

\section{Kohlenhydratlösungen}

Mengen unter $20 \mathrm{~g} \mathrm{KH/l}$ sind kaum wirksam, bei mehr als $90 \mathrm{~g} \mathrm{KH/l} \mathrm{kommt} \mathrm{es} \mathrm{leicht} \mathrm{zu} \mathrm{Unverträg-}$ lichkeiten. Belastungsintensitäten $>70 \% \mathrm{VO}_{2} \max$ beeinflussen die Magenentleerungsrate negativ. Wird das Trinken bereits im Training „mittrainiert“, können Magenentleerungsrate und Verträglichkeit auch unter Wettkampfbedingungen verbessert werden [21].

\section{Die Sport-Mineralstoffe Calcium und Magnesium}

Bei sportlichen Aktivitäten gehen über den Ganzkörperschweiß durchschnittlich ca. 40 mg Calcium und 20 mg Magnesium pro Liter verloren (s. o. \ Tab. 1) [19]. Sinnvollerweise wird der vollständige Ersatz der Wasserverluste nach der Aktivität daher mit dem Ersatz dieser Elektrolytverluste durch mineralstoffreiches Mineralwasser kombiniert. Mineralwässer mit einem nennenswerten Calciumund Magnesiumgehalt sowie einem Verhältnis dieser Mineralstoffe von 2:1 können einen Beitrag leisten, beide Elektrolyte zu ersetzen und minimieren das Risiko von Mineralstoffdysbalancen.

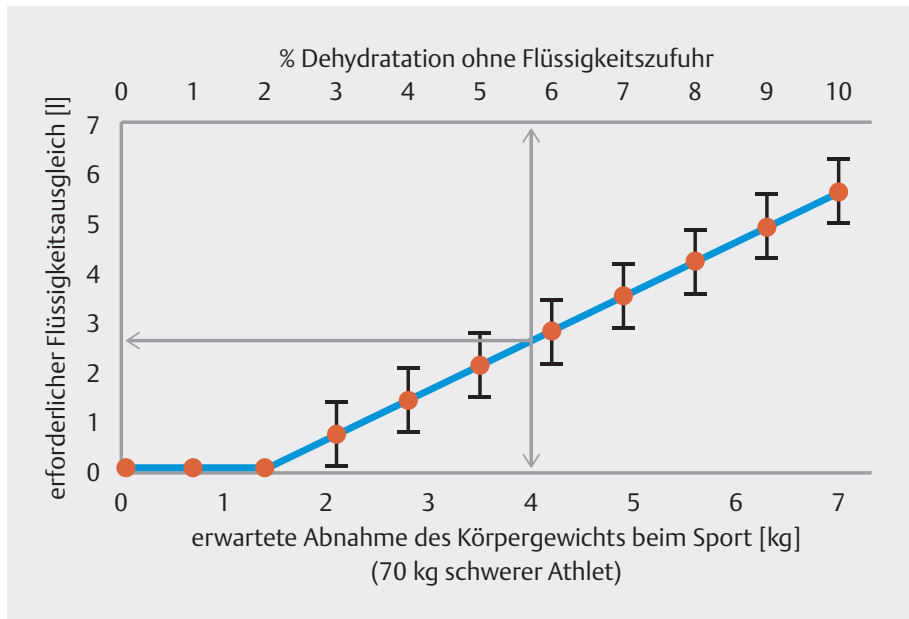

Abb. 2 Empfehlung zur Trinkmenge, um einen Verlust von > 2\% Körpergewicht als Wasser (Dehydratation) zu verhindern [23].

- Tab. 1 Durchschnittliche Schweißverluste bzw. Elektrolytgehalt im Ganzkörperschweiß und Elektrolytempfehlungen für ein Rehydratationsgetränk beim Marathon [19]. Daten aus 13 Studien.

\begin{tabular}{|l|l|l|l|l|l|}
\hline & \multicolumn{3}{|l}{ Elektrolyt [mg/l] } & & \\
\hline & $\mathrm{Cl}^{-}$ & $\mathbf{N a}^{+}$ & $\mathbf{K}^{+}$ & $\mathbf{C a}^{2+}$ & $\mathbf{M g}^{2+}$ \\
\hline $\begin{array}{l}\text { Durchschnitt, ca. } \\
\text { Absorption }\end{array}$ & 1015 & 750 & 170 & 40 & 20 \\
\hline $\begin{array}{l}\text { Praxisempfehlung** } \\
\text { für den Ausgleich }\end{array}$ & 100 & 100 & 100 & $30^{*}$ & $35^{*}$ \\
\hline
\end{tabular}

* Da die Resorptionsquote bei Calcium und Magnesium nur 30-35\% beträgt, muss die aufzunehmende Menge zum Ausgleich dreimal so hoch sein. ** Deutsches Institut für Sporternährung e. V.

\section{Merke \\ Ein Hydrogencarbonatgehalt $\left(\mathrm{HCO}_{3}{ }^{-}\right)>1000 \mathrm{mg} / \mathrm{l}$ wertet das Mineralwasser zusätzlich auf.}

Auch der Weltfußballverband FIFA empfiehlt den Konsum von Mineralwasser statt Leitungswasser wegen der Mineralisierung und des möglichen Gehaltes an unerwünschten Stoffen im sog. Stagnationswasser [14]. Gefährlich ist Stagnationswasser, wenn veraltete Wasserleitungsrohre verbaut sind oder sich Biofilme in den Leitungen bilden. Verunreinigungen durch Stoffe wie Blei können die Folge sein. Auch die Perlatoren der Trinkwasserhähne von Sporthallen, Trainingsplätzen und Fitness-Studios müssen regelmäßig getauscht oder zumindest gereinigt werden.

\section{Merke \\ Mineralstoffreiches Mineralwasser sollte beim Sport dem Trinkwasser vorgezogen werden.}

Mineralstoffreiches Mineralwasser ist ebenfalls als Regenerationsgetränk empfehlenswert. Trinkmengen, die ad- 
diert mit den während der Aktivität verzehrten Getränken die Schweißverluste zu mindestens $130 \%$ ersetzen, sind anzustreben. Lebensmittel wie Laugengebäck oder Salzstangen können den Natriumanteil beisteuern und sind darüber hinaus Lieferanten von schnell verfügbaren Kohlenhydraten zur Resynthese der entleerten muskulären Glykogenspeicher.

Für die Wiederauffüllung der Glykogenspeicher nach sehr langen und intensiven Trainingseinheiten wird auch Kalium benötigt. Fruchtsaftschorlen sowie alkoholfreies Bier und alkoholfreier Apfelwein sind kaliumreich und gute Kohlenhydratlieferanten. Sie genießen eine hohe geschmackliche Akzeptanz und sind daher gerade im Freizeit- und Breitensport praktikable Recovery-Getränke.

\section{KERNAUSSAGEN}

Sportliche Leistungen sind geknüpft an einen ausgeglichenen Flüssigkeitshaushalt und die Restitution von durch den Schweiß verlorenen Elektrolyten. Eine individuelle Trinkstrategie, die bereits in der Trainingsphase zum Einsatz kommt, unterstützt die Leistungsfähigkeit des Körpers und fördert nach dem Sport oder Wettkampf die Regeneration. Trinkempfehlungen richten sich an die individuellen physiologischen Voraussetzungen und berücksichtigen neben der Art und Dauer der sportlichen Leistung die Mineralstoffverluste durch den Schweiß, ggf. einen Ausgleich verbrauchter Kohlenhydrate sowie die aktuellen Wetterbedingungen.

\section{Interessenkonflikt}

Die Autoren geben folgende Interessenkonflikte an: Durchführung von ernährungswissenschaftlichen Studien zu den Themenbereichen Mineralwasser, Alkoholfreies Bier, Alkoholfreier Apfelwein, Vortragshonorare von HassiaMineralquellen, Gerolsteiner Sprudel, Bitburger Brauerei, Verband Deutscher Mineralbrunnen (VDM) sowie Beratertätigkeiten für den Verband Deutscher Mineralbrunnen/ Plattform Dialog Mineralwasser

\section{Autoren}

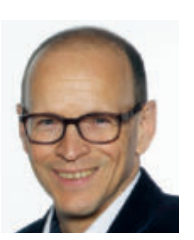

\section{Uwe Schröder}

Uwe Schröder ist Dipl.-Oecotrophologe. Er führt praxisorientierte Studien und das Sportler-Ernährungs-Coaching am Deutschen Institut für Sporternährung e. V. durch und berät Patienten des ambulanten Rehabilitationsbereichs der Sportklinik Bad Nauheim. Zudem ist er Lehrbeauftragter für „Sportlerernährung“ an der Hochschule Fulda.

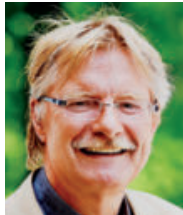

\section{Günter Wagner}

Günter Wagner ist als Dipl.-Oecotrophologe Mitglied des Vorstandes im Deutschen Institut für Sporternährung e. V. Im Rahmen der sportmedizinischen Betreuung der Sportkliniken Bad Nauheim und Frankfurt berät er Leistungs- und Freizeitsportler. Er ist Mitglied des wissenschaftlichen Beirats des VFED e. V. sowie Zweiter Vorsitzender der Gesellschaft für Gehirntraining (GfG) e. V., Ebersberg.

\section{Korrespondenzadresse}

Dipl. oec.-troph. Günter Wagner

Mitglied des Vorstandes

Deutsches Institut für Sporternährung e. V.

in der Sportklinik Bad Nauheim

In der Aue 30-32

61231 Bad Nauheim

www.dise.online

www.sportklinik-badnauheim.de

E-Mail: g.wagner@dise.online

\section{Literatur}

[1] Sawka M, Pandolf KB. Effects of body water loss on physiological function and exercise performance. In: Gisolfi C, Lamb DR, eds. Perspectives in Exercise Science and Sports Medicine. Carmel, CA, USA: Benchmark Press; 1990; 3: 1-38

[2] Cheuvront SN, Carter R, 3 rd, Sawka MN. Fluid balance and endurance exercise performance. Curr Sports Med Rep 2003; 2: 202-208

[3] Cheuvront SN, Kenefick RW, Montain S], Sawka MN. Mechanisms of aerobic performance impairment with heat stress and dehydration. J Appl Physiol 2010; 109: 1989-1995

[4] Sawka MN, Burke LM, Eichner ER et al. American College of Sports Medicine position stand. Exercise and fluid replacement. Med Sci Sports Exerc 2007; 39: 377-390

[5] McDermott BP, Anderson SA, Armstrong LE et al. National Athletic Trainers' Association Position Statement: Fluid replacement for the physically active. J Athl Train 2017; 52: 877-895

[6] Krabak B], Parker KM, DiGirolamo A. Exercise-associated collapse: Is hyponatremia in our head? PM R 2016; 8(Suppl. 3): S61-S68. doi:10.1016/j.pmrj.2015.10.002

[7] Lipman GS, Burns P, Phillips C et al. Effect of sodium supplements and climate on dysnatremia during ultramarathon running. Clin J Sport Med 2020. doi: 10.1097| JSM.0000000000000832

[8] McDermott BP, Anderson SA, Armstrong LE et al. National Athletic Trainers' Association Position Statement: fluid replacement for the physically active. J Athl Train 2017; 52: 877-895

[9] Kenefick RW, Cheuvront SN. Physiological adjustments to hypohydration: Impact on thermoregulation. Auton Neurosci 2016; 196: 47-51

[10] Trangmar S], Gonzalez-Alonso J. New insights into the impact of dehydration on blood flow and metabolism during exercise. Exerc Sport Sci Rev 2017; 45: 146-153

[11] Trangmar SJ, Gonzalez-Alonso J. Heat, hydration and the human brain, heart and skeletal muscles. Sports Med 2019; 49: $69-85$ 
[12] Lehrl S, Wagne, G, Schröder U. Die optimale Trinkmenge für die maximale geistige Leistungsfähigkeit. Der Allgemeinarzt 1999; 21: 664-667

[13] Schmitz J, Lehrl S, Schröder U, Wagner G. Einfluss von Dehydratation auf die kognitive Leistungsfähigkeit im Rahmen der Rosbacher Trinkstudie (RTS) 1-4, 40. Wissenschaftlicher Kongress der Deutschen Gesellschaft für Ernährung e. V., Potsdam; 2003

[14] Culvenor AG. FIFA Diploma in Football Medicine: free knowledge from expert clinicians to improve sports medicine care for all football players. Br J Sports Med 2017; 51: 1338-1339. doi:10.1136/bjsports-2016-096662

[15] Baker LB, Barnes KA, Anderson ML et al. Normative data for regional sweat sodium concentration and whole-body sweating rate in athletes. J Sports Sci 2016; 34: 358-368

[16] Tyler C], Reeve T, Hodges C], Cheung SS. The effects of heat adaptation on physiology, perception, and exercise performance in the heat: A meta-analysis. Sports Med 2016; 46: 1699-1724

[17] Armstrong LE, Casa DJ. Methods to evaluate electrolyte and water turnover of athletes. Athl Train Sports Health Care; 2009; 1: 169-179. doi:10.3928/19425864-20090625-06

[18] Thomas DT, Erdman KA, Burke LM. Position of the Academy of Nutrition and Dietetics, Dietitians of Canada, and the American College of Sports Medicine: Nutrition and Athletic Performance. J Acad Nutr Diet 2016; 116: 501-528

[19] Brouns F, Saris W, Schneider H. Rationale for upper limits of electrolyte replacement during exercise. Int J Sport Nutr 1992; 2: 229-238
[20] EFSA Panel on Dietetic Products, Nutrition and Allergies (NDA); Scientific Opinion on the substantiation of health claims related to carbohydrate-electrolyte solutions and reduction in rated perceived exertion/effort during exercise (ID 460, 466, 467, 468), enhancement of water absorption during exercise (ID 314, 315, 316, 317, 319, 322, 325, $332,408,465,473,1168,1574,1593,1618,4302,4309$ ), and maintenance of endurance performance (ID 466, 469) pursuant to Article 13(1) of Regulation (EC) No 1924/2006. EFSA Journal 2011; 9: 2211

[21] Horner KM, Schubert MM, Desbrow B et al. Acute exercise and gastric emptying: A meta-analysis and implications for appetite control. Sports Med 2015; 45: 659-678

[22] EFSA NDA Panel, Turck D, Bresson J-L, Burlingame B et al. Scientific opinion on the carbohydrate solutions and contribute to the improvement of physical performance during a high-intensity and long-lasting physical exercise: evaluation of a health claim pursuant to Article 13(5) of Regulation (EC) No 1924/2006. EFSA Journal 2018; 16: 5191

[23] Belval LN, Hosokawa Y, Casa DJ et al. Practical hydration solutions for sports. Nutrients 2019; 11: 1550

Bibliografie

DOI https://doi.org/10.1055/a-0971-0665

Ernährung \& Medizin 2020; 35: 10-15

(c) Georg Thieme Verlag KG Stuttgart · New York ISSN 1439-1635 